\section{Osteodistrofia fibrosa em canino idoso secundário à doença renal crônica: relato de caso}

\author{
Fibrous osteodystrophy in elderly canine secondary to chronic \\ kidney disease: case report
}

Rosemar de Almeida Freitas ${ }^{1 *}$, Aline Fernandes Porto ${ }^{2}$, Mário dos Santos Filho ${ }^{3}$ \& Jonimar Pereira Paiva ${ }^{4}$

'Médica veterinária, MSc, Docente da Escola de Veterinária. Universidade Castelo Branco - UCB, Rio de Janeiro, RJ, Brasil 2Médica veterinária, Autônoma. Rio de Janeiro, RJ, Brasil

${ }^{3}$ Médico veterinário, Mestrando. Programa de Pós-graduação em Medicina Veterinária, Ciências Clínicas, Instituto de Veterinária - IV, Universidade Federal Rural do Rio de Janeiro - UFRRJ, Seropédica, RJ, Brasil

${ }^{4}$ Médico veterinário, DSc, Docente. Departamento de Medicina e Cirurgia Veterinária, Instituto de Veterinária - IV, Universidade Federal Rural do Rio de Janeiro - UFRRJ, Seropédica, RJ, Brasil

\section{Resumo}

A osteodistrofia renal ou osteodistrofia fibrosa é uma complicação decorrente do hiperparatireoidismo secundário à doença renal crônica, que pode estar presente desde os estágios iniciais. A osteodistrofia é um distúrbio osteopênico com proliferação do tecido conjuntivo fibroso, mais evidente nos ossos mandibulares e maxilares, que acomete frequentemente animais jovens eé menos frequente em animais idosos. O presente relato tem como objetivo principal descrever o caso de um canino fêmea com nove anos de idade, sem raça definida, com hiperparatireoidismo secundário a doença renal crônica que evoluiu para uma osteodistrofia fibrosa renal.

Palavras-chave: cálcio, fósforo, azotemia, rins.

\begin{abstract}
Renal osteodystrophy or fibrous osteodystrophy is a complication of hyperparathyroidism secondary to chronic kidney disease, which may be present from the earliest stages. Osteoarthritis is an osteopenic disorder with the proliferation of fibrous connective tissue, most evident in the mandibular and maxillary bones, which affects young animals more frequently and is less frequent in older animals. The present report has as main objective to describe the case of an elderly female canine, with no defined race, with hyperparathyroidism secondary to a chronic renal disease that evolved to renal fibrous osteodystrophy.
\end{abstract}

Keywords: calcium, phosphor, azotemia, kidneys.

\section{Introdução}

O hiperparatireoidismo secundário renal (HSR) é uma complicação comum na doença renal crônica (DRC), em virtude de, dentre as funções exercidas pelos rins, está a excretora e endócrina, que estarão comprometidas na presença da DRC (Alves et al., 2014). O HSR já pode estar presente desde o estágio II da DRC (Rusenov, 2010; Chew et al., 2012), pois a redução da função renal promove diminuição na produção de calcitriol e retenção de fósforo, os quais contribuem para o surgimento e perpetuação do HSR (Custódio et al., 2013).

O HSR é uma síndrome complexa e multifatorial que envolve mudanças nos níveis circulantes de cálcio, paratormônio (PTH), fósforo e 1,25-diidroxicolecalciferol (calcitriol) (Polzin et al., 2009; Stillion \& Ritt, 2009).

Ao PTH, hormônio produzido pelas glândulas paratireoides, cabe à função de manter as concentrações de cálcio extracelular (cálcio ionizável) dentro de níveis séricos adequados (Parker et al., 2015). O contrário também ocorre, onde a própria concentração do cálcio ionizável regula a secreção de PTH fazendo, dessa forma, o processo chamado de feedback. Sendo assim, quando há aumento dos níveis séricos de cálcio ionizável, a produção de PTHé reduzida (Rodríguez, 1995) e na presença de uma hipocalcemia ionizada, as glândulas paratireóides iniciam a secreção de PTH (Chew et al., 2012) e este por sua vez, mobilizará cálcio dos ossos. Em contrapartida, o calcitrol estimula a absorção de cálcio pelo intestino (Holowaychuk, 2013). Como o calcitrol é produzido pelo rim, na DRC seus níveis estarão reduzidos (Polzin et al., 2009), contribuindo ainda mais para a hipocalcemia, e aumentando indiretamente a liberação do PTH(Custódio et al., 2013).
Como citar: Freitas, R. A., Porto, A. F., Santos Filho, M., \& Paiva, J. P. (2017). Osteodistrofia fibrosa em canino idoso secundário à doença renal crônica: relato de caso. Brazilian Journal of Veterinary Medicine, 39(3), 215-220. doi: 10.29374/2527-2179. bjvm013917

Fonte de financiamento: MSF é bolsista CAPES.

Conflito de interesses: Os autores declaram não haver conflitos de interesse que precisam ser informados.

Recebido: Novembro 14, 2016. Aceito: Março 19, 2017

O estudo foi realizado na Clínica Escola Paulo Alfredo Gissoni, Universidade Castelo Branco - UCB, Rio de Janeiro, RJ, Brasil.

\section{*Correspondência}

Rosemar de Almeida Freitas

Universidade Castelo Branco - UCB

Avenida Brasil, 9727, Penha

CEP 21012-351 - Rio de Janeiro (RJ), Brasil

E-mail: rosemar@castelobranco.br
Copyright Freitas et al. Este é um artigo publicado em acesso aberto (Open Access) sob a licença Creative Commons Attribution Non-Commercial, que permite uso, distribuição e reprodução em qualquer meio, sem restrições desde que sem fins comerciais e que o trabalho original seja corretamente citado. 
O PTH é o tipo de toxina urêmica que exerce efeitos adversos no cérebro, coração, medula óssea e tecidos moles; além de ter um importante papel no desenvolvimento da osteodistrofia fibrosa (Chew et al., 2012).

A constante secreção do PTH presente nos quadros de DRC pode levar a hiperplasia da glândula paratireóide. Além de levar a quadros de desmineralização óssea e mineralização de tecidos moles (Lazaretti et al., 2006).

A manifestação clínica mais importante associado ao HSR é a osteodistrofia fibrosa ou "mandíbula de borracha" (Tilley \& Smith Junior, 2008), caracterizada por aumento de volume na região mandibular, podendo ser observado por meio do exame físico, o tecido ósseo de consistência macia e flexível (Stillion \& Ritt, 2009).

Para o diagnóstico de HSR, as concentrações séricas de PTH intacto devem ser mensuradas de forma seriada, pois uma única mensuração com valores dentro do padrão de normalidade não exclui a possibilidade da ocorrência da doença (Chew et al., 2012). Exames radiográficos podem revelar baixa densidade óssea, perda de lâmina dura em torno dos dentes e desmineralização da maxila e da mandíbula (Headley et al., 2008; Rusenov, 2010).

Por se tratar de um distúrbio complexo, o tratamento deve ser ajustado para cada paciente (Carvalho et al., 2012), e envolve o controle do fósforo sérico por meio da dieta e de quelantes, além da possível suplementação de Vitamina D (Parker et al., 2015).

O prognóstico clínico pode variar de reservado a ruim (Tilley \& Smith Junior, 2008; Stillion \& Ritt, 2009) e geralmente culmina com a eutanásia do paciente (Vanbrugghe et al., 2011).

\section{Histórico}

Paciente canino, fêmea, esterilizada, sem raça definida, de nove anos de idade e pesando $12 \mathrm{~kg}$ foi encaminhada para acompanhamento nefrológico na Clínica Escola Paulo Alfredo Gissoni da Universidade Castelo Branco - UCB, com histórico de doença renal crônica de evolução há aproximadamente seis meses, mas clinicamente estável. Na ocasião, a paciente encontrava-se em estágio 3 da DRC (creatinina: 4.5mg/dL - Ref.: 1.4mg/dL; uréia: 260mg/dL - Ref.: 20-60mg/dL) e com uma proteinúria persistente de 3,2 (Ref.: 0,2-0,5), sem sedimento urinário ativo e/ou infecção bacteriana associada. As concentrações séricas de sódio, potássio, albumina, cálcio total e iônico encontravam-se dentro dos valores de referência. No entanto, o fósforo encontrava-se aumentado com valor de $10 \mathrm{mEg} / \mathrm{L}$ (Ref.: 2,6-6,2mEg/L). O exame hematológico apresentava discreta anemia normocítica normocrômica, com hematócrito de 30\% (Ref.: 37-55\%), hemoglobina 8.5g/dL (Ref.: 14-18g/dL) e hemácias de 6.3 (Ref.: 5.5-8.5 x10\%/ $\mu \mathrm{L}$ ). Não foram observadas alterações na série branca.

O exame ultrassonográfico da região abdominal mais recente, revelava rins em topografia habitual, com dimensões aumentadas, formato anatômico preservado, contorno regular e a relação corticomedular preservada. A camada cortical apresentava aumento da sua ecogenicidade e aspecto grosseiro, mais evidenciado em rim esquerdo. Foi visualizada ainda, na ocasião, a presença de uma imagem ecóica margeando a cortical e medular, sinal medular sugerindo nefropatia crônica.

Antes de iniciar o exame físico da paciente, foi mensurada sua pressão arterial sistólica sistêmica, sendo constatada hipertensão, em valores de $180 \mathrm{mmHg}$ (Ref: $\leq 150 \mathrm{mmHg}$ ), classificada como risco alto de lesão em órgão alvo (Brown et al., 2007).

Ao examiná-la, foi constatado que paciente se encontrava em boa condição corpórea (score 4 - Ref::1/9), mucosas normocoradas, normohidratada, ausência de desconforto à palpação abdominal, doença periodontal grave, frouxidão dos dentes, halitose urêmica, ausculta pulmonar sem alterações e ausculta cardíaca relevando sopro sistólico em foco mitral. Sem mais alterações de exame físico dignas de nota.

Foi então prescrito fluidoterapia com solução Ringer com lactato na dose de $30 \mathrm{~mL} / \mathrm{Kg}$ por via endovenosa, durante 5 dias seguidos associado ao cloridrato de benazepril (0.5mg/Kg/via oral $/ 24 \mathrm{~h} / 7 \mathrm{dias})$. Mantida a ração terapêutica renal e o hidróxido de alumínio que a paciente já estava fazendo uso. Foi então solicitado ecocardiograma com avaliação cardiológica, novo exame de urina com relação proteína/creatinina urinária (para monitoramento da proteinúria), exame radiográfico da região craniana nas posições latero-lateral e oblíqua (a fim de pesquisa de alterações periodontais, que poderiam agravar a disfunção renal), dosagem sérica de PTH intacto e de vitamina $\mathrm{D}_{3}$ (1,25-dihidroxicocicalciferol = calcitriol). 
Os resultados dos exames solicitados demonstraram a existência de doença valvar degenerativa crônica de mitral em estágio B2 e no exame de urina a permanência de proteinúria com discreta redução para 2.8, não demonstrando melhora após a introdução do cloridrato de benazepril.

O exame radiográfico revelou maxila e ramo mandibular com perda moderada de densidade óssea difusa; elementos dentais bem visualizados, com periferias apicais mais definidas e destacadas que o habitual por conta da perda de densidade alveolar; aspecto radiográfico "fibroso" sobreárea de plano nasal (Figura 1); e, sínfise mentoniana simétrica e com sinal de estabilidade (Figura 2). Desta forma, as imagens são compatíveis com quadro de HSR de apresentação radiográfica moderada, sem aumento facial.

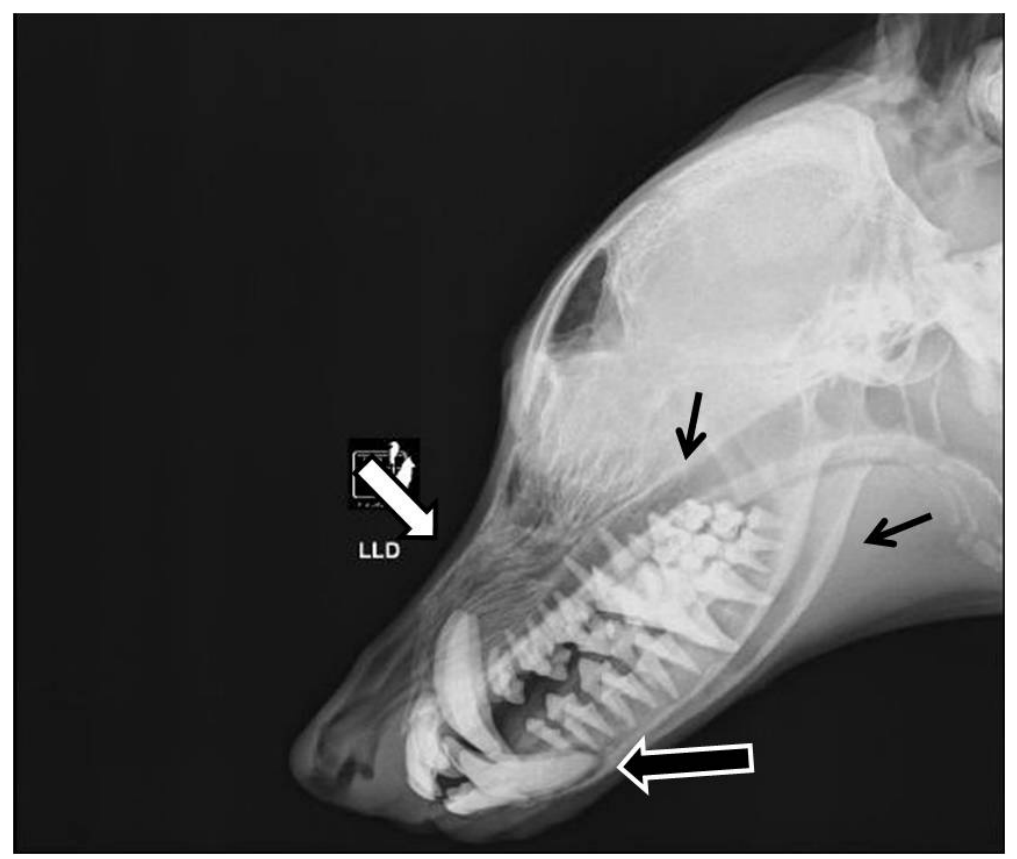

Figura 1. Imagem da maxila e do ramo mandibular com perda moderada de densidade óssea difusa (setas finas), periferia apical, dos dentes, mais definidos e destacados por conta da perda de densidade alveolar (contorno de seta) e aspecto radiográfico "fibroso" sobre área de plano nasal (seta preenchida).

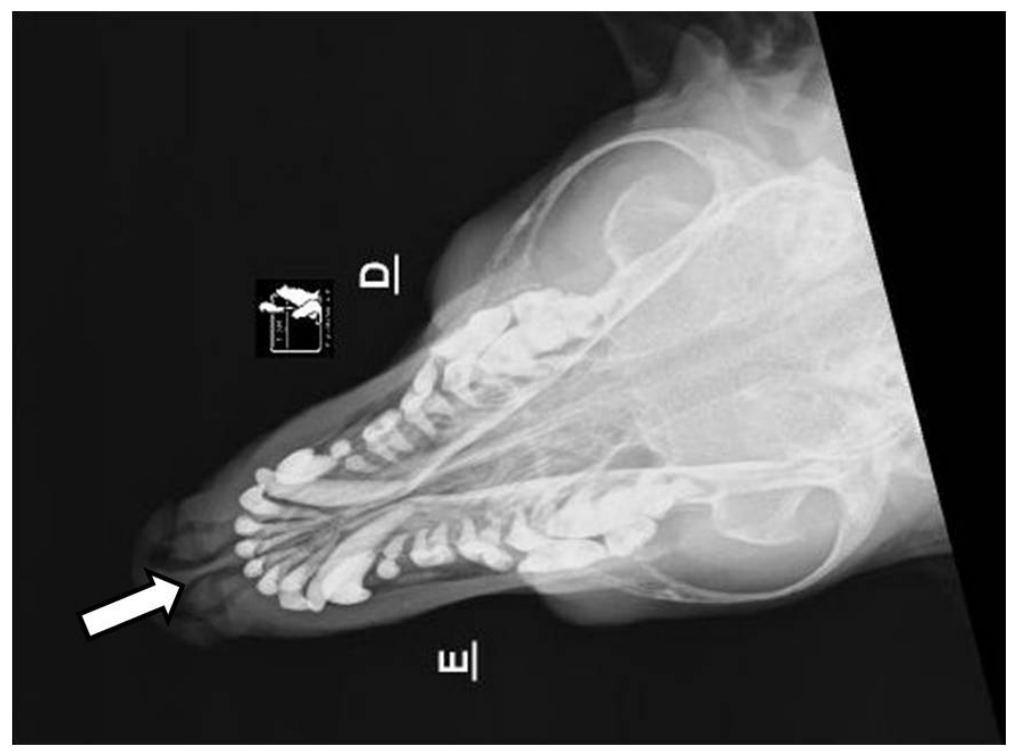

Figura 2. Sínfise mentoniana simétrica e com sinal de estabilidade (seta preenchida). D- antímero direito; E- antímero esquerdo. 
Não houve alterações nas concentrações séricas de PTH intacto (6,2 pg/mL - Ref.: 5-25 pg/mL), tão pouco nas concentrações de vitamina $\mathrm{D}_{3}$ (147pg/mL - Ref.: 103-203pg/mL).

Durante a realização dos exames, o paciente descompensou e iniciou um quadro urêmico com anorexia, perda de peso, prostração, halitose, náuseas, êmese e aumento da azotemia (ureia: 360 e creatinina: 10), precisando ser hospitalizada. Entretanto, como não houve melhora nos sintomas após 72 horas de hospitalização e dado os resultados dos exames laboratoriais e de imagem, a tutora optou pela eutanásia da paciente.

\section{Discussão}

A osteodistrofia fibrosa não é uma manifestação comum do hiperparatireoidismo secundário renal em pacientes idosos (Alves et al., 2014), sendo a sua ocorrência mais relatada na literatura, em animais jovens em fase de crescimento ósseo (Rusenov, 2010). No entanto, é esperada a sua ocorrência uma vez que na doença renal crônica a lesão renal estrutural e/ou funcional são irreversíveis e progressivas (Jacob et al., 2005; Bartges, 2012; Chew et al., 2012).

Os achados radiográficos do presente relato indicam fortemente o processo de osteodistrofia fibrosa e uma vez associados aos achados de exame clínico e laboratoriais direcionam para o hiperparatireoidismo secundário à perda crônica da função renal, principalmente no que tange a desmineralização óssea provocada pela remoção de cálcio do tecido ósseo estimulada pelo paratormônio e posterior substituição por tecido conjuntivo fibroso (Alves et al., 2014).

No presente relato, foi solicitado o exame radiográfico do crânio como forma de se avaliar a desmineralização óssea, mesmo sem achados de exame clínico que a sugerissem. Esta conduta possibilitou o diagnóstico da osteodistrofia diante de valores normais tanto do PTH intacto, quanto do calcitriol (Brito Galvao et al., 2013). Tal achado pode sugerir que diante do diagnóstico de DRC já em quadros de hiperfosfatemia ou não, a osteodistrofia deve ser buscada para melhor entendimento da evolução da doença, principalmente em função dos diferentes mecanismos e motivos que podem fazer com que alguns exames laboratoriais se mantenham dentro da normalidade (Custódio et al., 2013).

A regulação do cálcio sérico é orquestrada pelo PTHe pelo calcitriol. Sendo assim, na ocorrência de hipocalcemia iônica, a secreção de PTH influenciará de forma direta na mobilização e remoção de cálcio dos ossos. (Holowaychuk, 2013). Tal fato justifica a ausência da hipocalcemia no presente relato, em virtude da atuação do PTH mobilizando esse o cálcio ósseo, e do momento de dosagem desses níveis séricos em um momento já compensatório dos níveis de cálcio no sangue. (Custódio et al., 2013).

O PTH atua nos túbulos contornados proximais dos rins, aumentando a reabsorção do cálcio, e no tecido ósseo, estimulando os osteoclastos a digerirem a matriz óssea e com isso aumentar e/ou normalizar os níveis de íons cálcio (Parker et al., 2015). Ele ainda estimula o aumento da excreção renal de fosfato e o aumento da liberação de cálcio e fosfato pelos ossos, o que retorna ao normal as concentrações séricas de fósforo e cálcio ionizado, fazendo-se compreender o porquê da normocalcemia. O PTH diminui a fração de reabsorção de fosfato no rim pela diminuição da reabsorção tubular máxima de fosfato. Esse efeito inicialmente contribui para manutenção do fosfato nos níveis normais e mais fósforo é excretado na urina. O limite dessa resposta compensatória é alcançado quando a taxa de filtração glomerular (TFG) declina para aproximadamente 15\% a 20\% do normal. Com o declínio contínuo desta taxa, inevitavelmente ocorre a hiperfosfatemia (Stillion \& Ritt, 2009; Parker et al., 2015; Chew et al., 2012).

Como para o diagnóstico preciso do HSR, as concentrações séricas de PTH devem ser mensuradas de forma seriada, o que não houve tempo de ser feito com a paciente em questão em decorrência da rápida evolução seguida de eutanásia, uma única mensuração do PTH pode revelar valores dentro do padrão de normalidade não excluindo a existência da doença (Stillion \& Ritt, 2009).

É descrito que a hiperfosfatemia reduz a concentração sérica de cálcio ionizado pelo efeito da Lei da Massa ([Ca]x[P]= constante). No entanto, acredita-se que o efeito dessa lei seja pequeno, pois é preciso uma mudança considerável na concentração de fósforo sérico para produzir uma mudança muito pequena na concentração sérica de cálcio ionizado (Chew et al., 2012).

A hipertensão sistêmica da paciente pode ser justificada pela existência de doença glomerular (Brown et al., 2007), como consequência do aumento da pressão hidrostática decorrente da 
diminuição da pressão oncótica associada à ativação do sistema renina-angiotensina-aldosterona (Pressler, 2011).

A proteinúria persistenteé considerada um fator de progressão da doença renal indicativa de lesão renal, estando a sua intensidade associada à uma lesão renal mais grave, como glomerulonefrite (Nabity et al., 2011).

Todavia o prognóstico destes pacientes é de reservado à ruim e normalmente culminam com eutanásia, como ocorrido neste caso, devido ao estágio avançado do HSR, bem como a ausência de resposta à terapêutica (Vanbrugghe et al., 2011).

\section{Conclusão}

O hiperparatireoidismo secundário é uma alteração sistêmica grave e quase sempre é diagnosticada tardiamente, o que dificulta o manejo terapêutico e faz com que seu prognóstico seja de reservado a ruim.

A osteodistrofia fibrosa deve ser alvo de investigação por meio do exame radiográfico, principalmente diante de valores normais nos níveis séricos de PTH e calcitriol, como forma de melhor compreensão da evolução do HSR.

\section{Referências}

Alves, M. A. M. K., Crivellenti, L. Z., \& Carvalho, M. B. (2014). Osteodistrofia fibrosa de origem renal em dois cães idosos: relato de caso. Revista Portuguesa de Ciências Veterinárias, 109, 51-56.

Bartges, J. W. (2012). Chronic kidney disease in dogs and cats. The Veterinary Clinics of North America. Small Animal Practice, 42(4), 669-692, vi. http://dx.doi.org/10.1016/j.cvsm.2012.04.008. PMid:22720808.

Brito Galvao, J. F., Nagode, L. A., Schenck, P. A., \& Chew, D. J. (2013). Calcitriol, calcidiol, parathyroid hormone, and fibroblast growth factor-23 interactions in chronic kidney disease. Journal of Veterinary Emergency and Critical Care, 23(2), 134-162. http://dx.doi.org/10.1111/vec.12036. PMid:23566108.

Brown, S., Atkins, C., Bagley, R., Carr, A., Cowgill, L., Davidson, M., Egner, B., Elliott, J., Henik, R., Labato, M., Littman, M., Polzin, D., Ross, L., Snyder, P., Stepien, R., \& American College of Veterinary Internal Medicine (2007). Guidelines for the identification, valuation, and management of systemic hypertension in dogs and cats. Journal of Veterinary Internal Medicine, 21(3), 542-558. http://dx.doi.org/10.1111/j.1939-1676.2007.tb03005.x. PMid:17552466.

Chew D. J., Dibartola S. P., Chew D. J., Dibartola S. P. \& Schenck P. A. (2012). Urologia e Nefrologia do Cão e do Gato (2. ed.). Rio de Janeiro: Elsevier.

Carvalho, A. B., Gueiros, A. P. S., Gueiros, J. E. B., Neves C. L., Karohl, C., Sampaio E., Canziani, M. E., Custódio M. R., Moysés R. M. A., Barreto F., \& Jorgetti, V. Adendo das Diretrizes Brasileiras de Prática Clínica para o Distúrbio Mineral e Ósseo na Doença Renal Crônica. Jornal Brasileiro de Nefrologia, 34(2):199-205, 2012.

Custódio, M. R., Canziani, M. E. F., Moyses, R. M. A., Barreto, F. C., Neves, C. L., Oliveira, R. B., Karohl, C., Sampaio, E. A., Gueiros, J. E. B., Jorgetti, V., \& Carvalho, A. B. (2013). Protocolo clínico e diretrizes terapêuticas para o tratamento do hiperparatireoidismo secundário em pacientes com doença renal crônica. Jornal Brasileiro de Nefrologia, 35(4), 308-322.

Headley, S. A., Veltrini, V. C., Fuck, E. J., Fuck, E. T., Curti, C. E., Bettini, C. M., Bracarense, A. P. F. R. L., \& Reis, A. C. F. (2008). Lesões orais associadas ao hiperparatireoidismo renal secundário em um Buldogue Inglês. Semina: Ciências Agrárias, 29(2), 407-412. http://dx.doi.org/10.5433/1679-0359.2008v29n2p407.

Holowaychuk, M. K. (2013). Hypocalcemia of critical illness in dogs and cats. The Veterinary Clinics of North America. Small Animal Practice, 43(6), 1299-1317, vi-vii. http://dx.doi.org/10.1016/j.cvsm.2013.07.008. PMid:24144092.

Jacob, F., Polzin, D. J., Osborne, C. A., Neaton, J. D., Kirk, C. A., Allen, T. A., \& Swanson, L. L. (2005). Evaluation of the association between initial proteinuria and morbidity rate or death in dogs with naturally occurring chronic renal failure. Journal of the American Veterinary Medical Association, 226(3), 393-400. http://dx.doi. org/10.2460/javma.2005.226.393. PMid:15702689.

Lazaretti, P., Kogika, M. M., Hagiwara, M. K., Lustoza, M. D., \& Mirandola, R. M. S. (2006). Concentração sérica de paratormônio intacto em cães com insuficiência renal crônica. Arquivo Brasileiro de Medicina Veterinária e Zootecnia, 58(4), 489-494. http://dx.doi.org/10.1590/S0102-09352006000400006.

Nabity, M. B., Lees, G. E., Dangott, L. J., Cianciolo, R., Suchodolski, J. S., \& Steiner, J. M. (2011). Proteomic analysis of urine from male dogs during early stages of tubulointerstitial injury in a canine model of the progressive glomerular disease. Veterinary Clinical Pathology, 40(2), 222-236. http://dx.doi.org/10.1111/j.1939-165X.2011.00307.x. PMid:21446987.

Parker, V. J., Gilor, C., \& Chew, D. J. (2015). Feline Hyperparathyroidism: Pathophysiology, diagnosis, and treatment of primary and secondary disease. Journal of Feline Medicine and Surgery, 17(5), 427-439. http://dx.doi. org/10.1177/1098612X15581134. PMid:25896242. 
Polzin, D. J., Ross, S., \& Osborne, C. A. (2009). Calcitriol. In J. D. Bonagura \& D. C. Twedt (Eds.), Current Veterinary Therapy XIV, Small Animal Practice (pp. 892-895). St. Louis: WB Saunders.

Pressler, B. (2011). Nephrotic syndrome. In J. Bartges \& D.J. Polzin(Eds.), Nephrology and Urology of Small Animals. (1st ed., pp. 415-421). Ames: Wiley-Blackwell.

Rodríguez, M. (1995). Etiopatogenia del hiperparatireoidismo secundario: factores que afectan a la secreción de PTH. Nefrologia, 15, S25-S3O.

Rusenov, A. (2010). A clinical case of secondary hyperparathyroidism in a four-month-old Pug puppy. Revue de Medecine Veterinaire, 161(12), 570-573.

Stillion, J. R., \& Ritt, M. G. (2009). Renal secondary hyperparathyroidism in dogs. Compendium (Yardley, PA), 31(6), E8-E11. PMid:19746344.

Tilley, L. P., \& Smith Junior, F. W. K. (2008). Consulta Veterinária em 5 Minutos - Espécies Canina e Felina (3. ed.) Barueri: Manole.

Vanbrugghe, B., Blond, L., Carioto, L., Carmel, E. N., \& Nadeau, M. E. (2011). Clinical and computed tomography features of secondary renal hyperparathyroidism. The Canadian Veterinary Journal. La Revue Veterinaire Canadienne, 52(2), 177-180, quiz 180. PMid:21532826. 